\author{
Military Technical College \\ Kobry El-Kobbah, \\ Cairo, Egypt.
}

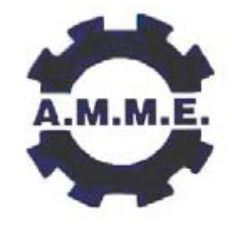

\title{
MODELISATION OF THERMOELASTIC LINEAR BEHAVIOUR OF TUNGSTEN FILAMENTS IN TWIN HELICAL SPRING CONFIGURATION
}

\author{
BENGHANEM ${ }^{(1)} N$., KAOUA ${ }^{(2)}$ S., BECHEUR ${ }^{(3)} A$. and $A Z Z A Z Z^{(4)} M$.
}

\begin{abstract}
By trying to increase the luminosity or the life expectancy of an incandescent lamp, an idea consists of a series of successive rolling up of the tungsten filament. This gives geometrical configurations of filaments named simple, twin, or triple helical spring, etc. In this paper, we will limit only to the case of the twin helical spring filament. Under the combined effects of the high temperature and the stresses induced by its own weight, the filament creeps by becoming plastically deformed in a permanent and a continuously manner, until the rupture. The theoretical study of the behaviour of these filaments needs the knowledge of internal loads distribution, deformations modes, and their evolutions. The present work consists on an elastic structural analysis which must be the first step. Since the objective is also to study the influence of the filament self weight solicitation, the orientation with respect to gravitational direction should be taken into account. Numerical results are obtained by finite element method. They showed a negligible influence of the solicitations due to the variation of the temperature with respect to those of the self weight, and a relative sensitivity to the position changes. For the case of a vertical position, an analytical method has also been developed. Because of some difficulties, the comparison between the numerical and analytical results could not be carried out directly. However, the analytical approach proved to be qualitatively useful for the analysis of numerical results.
\end{abstract}

\section{KEY WORDS}

Twin helical spring filament, self weight, temperature, position, finite elements

(1) Assistant professor, Faculté des sciences de la nature et de la vie. Université de Bejaia 06000 ALGERIA

(2) Assistant professor, Faculté de génie Mécanique et de génie des procédés USTHB ALGER16000 ALGERIA

(3) Assistant professor, Laboratoire de Technologie des Matériaux et de Génie des Procédés LTMGP. Université de Bejaia 06000 ALGERIA

(4) Professor, Faculté de génie Mécanique et de génie des procédés USTHB ALGER16000 ALGERIA 


\section{INTRODUCTION}

The theoretical study of the behaviour of tungsten filaments needs the knowledge of internal loads distribution, deformations modes, and their evolutions within creep. The present work consists on an elastic structural analysis which must be the first step. In this framework, we will try to give some elements of answers to practical questions linked to the initial phase which is governed by an elastic behaviour. These answers should also be used as initial conditions for other studies such as plasticity, damage mechanics or creeps. They consist in several points such as:

- the initial distribution of the internal loads before putting under tension of the filament (or under the isolated effect of the self weight filament);

- the influence of the temperature with respect to the self weight or in other words, the contribution of the temperature variation to modify the internal loads distribution and intensities;

- the influence of the orientation (or position) with respect to gravitational direction

- and the influence of the inclinations of the whorls on the internal distribution of forces and moments

To our knowledge, for the modelisation of the twin helical spring filament, there are no available contributions in the literature. For the analysis of the behaviour with temperature, the theoretical contributions are also rare. For the mechanical aspect (without temperature), only simple helical springs have analytical and numerical contributions.

To obtain sufficiently reliable answers, the study should be undertaken both in an analytical and a numerical way. In this paper, we present an analytical method which we have developed for the twin helical spring filament in a vertical position in Benghanem et al, [01-02]. This analytical method is based on the principles of the vectorial analysis using transformations, and the theories of strength of materials (Timoshenko, [03]).

The numerical way will consists on the use of the finite element method. In deed, the analytical calculations for several cases such as the horizontal position, load cases relative to the effects of the variation in the temperature and the effect of the creep, prove to be complex and difficult. It comes then to mind the idea of the modelling by finite elements. This not only makes possibility to exceed the difficulty of geometrical descretisation but offers especially various possibilities of coupling such as thermoelasticity, thermo-plasticity, thermo-élasto-visco-plasticity, damage thermo-elasticity, etc.

On the other hand, we note that during the lamp working, the filament can be in various positions: vertical, horizontal, or inclined. To study the influence of the filament self weight solicitation, the orientation (or position) with respect to gravitational direction should be taken into account. To simplify, we will consider only the two principal positions: vertical and horizontal. The study of the vertical position will be carried out jointly in an analytical and a numerical way by the finite elements method. However, because of its complexity, horizontal position will only be studied by numerical finite element method. 
Moreover, the analysis will be static and under the assumptions of small deformations coupled with linear thermo-elastic behaviour. A presentation of the developed method for analytical study of vertical Twin Helical Spring (THS) filament will be shown. It will be followed by the numerical modelling of the twin (THS) helical spring filament configuration. This will be performed by the use of the two nodes three dimensional frame finite elements. The effects of the variation in the temperature will be numerically taken into account, but only in the linear elastic domain. This means that Young modulus, thermal dilatation coefficient and the yield stress will be assumed constant and independent of the temperature. This is not really the case (Tietz and Wilson, [04]) (Whittenberg and Nathal,[05]). Their taking into account will imply a non material linearity which must be the subject of future studies.

\section{ANALYTICAL STUDY OR A DEVELOPED METHOD FOR TWIN HELICAL SPRING FILAMENT CASE}

The manufacturing process of the twin helical spring filament needs two successive operations. The first rolling up operation gives a simple helical spring filament with small whorls named as the primary whorls with $R_{1}$ radius and $\alpha_{1}$ angle of inclination. The second consists in a rolling up of the SHS filament obtained previously. It will give a twin helical spring filament (figure 01) with two categories of whorls: the secondary whorl (with $R_{2}$ radius and $\alpha_{2}$ angle of inclination) being biggest than the primary.

Filament (THS) assembled horizontally in an electric bulb will present deflection initially. This makes the analytical study complex and difficult. To simplify; we undertake this analytical study on a filament whose position is supposed to be vertical and whose lower extremity is free and subjected to a vertical and punctual traction force $\vec{T}$. In order to represent correctly the self weight (figure 01 ), we assume that the force $\vec{T}$ is taken by the vertical and symmetrical $\overrightarrow{\mathrm{K}}$ axis (figure $02 \mathrm{a}$ ).

\section{The Coordinate Systems Definition}

The method developed is based on the adequate definition of the coordinate systems below and on the successive transformations from the $(O, \vec{i}, \vec{j}, \vec{k})$ system to the final $\left(\mathrm{O}^{\prime \prime}, \overrightarrow{\mathrm{f}}, \overrightarrow{\mathrm{d}}, \overrightarrow{\mathrm{e}}\right)$ system (figure 02). The forces and moments components will be determined by using matrix transformations and vectorial cross products. These final expressions will allow to compute and to analyse the internal forces and moments distributions.

We give in figure 02 the definition of local coordinate systems necessary to carry out the successive transformations. Such as they are represented, we can define by $\left(\mathrm{O}, \overrightarrow{\mathrm{i}}, \overrightarrow{\mathrm{j}}^{\prime}, \overrightarrow{\mathrm{k}}^{\prime}\right)$, the coordinate system linked to the plan of the secondary whorl (or spiral), by $\left(\mathrm{O}^{\prime}, \overrightarrow{\mathrm{i}}^{\prime \prime}, \overrightarrow{\mathrm{j}}^{\prime \prime}, \overrightarrow{\mathrm{k}}^{\prime \prime}\right)$ the coordinate system linked to the plan of the primary whorl and by $\left(\mathrm{O}^{\prime \prime}, \overrightarrow{\mathrm{f}}, \overrightarrow{\mathrm{d}}, \overrightarrow{\mathrm{e}}\right)$, the coordinate system linked to the cross section of the wire. The $\alpha_{1}$ and $\alpha_{2}$ angles (figure 02) represent the inclinations of the primary and secondary whorls which rolling up radii are $R_{1}$ and $R_{2}$ respectively. The $\varphi$ angle allows covering from 0 to $2 \pi$ all cross sections of a primary whorl. 


\section{Internal Forces and Moments Distributions}

On the local coordinate system $\left(\mathrm{O}^{\prime \prime}, \overrightarrow{\mathrm{f}}, \overrightarrow{\mathrm{d}}, \overrightarrow{\mathrm{e}}\right)$ linked to the cross section of the wire and located by the angles $\alpha_{1}, \alpha_{2}$ and $\varphi$, we will compute the applied resultant force $\vec{R}\left(V_{f}, V_{d}, N_{e}\right)$ and the resultant moment $\vec{M}_{\vec{T}}\left(M_{f}, M_{d}, M_{e}\right)$ components. $V_{d}$ and $V_{f}$ are the shear forces acting respectively on $\vec{d}$ and $\vec{f}$ axes. $N_{e}$ is the normal force on the $\vec{e}$ axis. $M_{d}$ and $M_{f}$ are the bending moments acting over $\vec{d}$ and $\vec{f}$ axes. $M_{e}$ is the torque moment on the $\overrightarrow{\mathrm{e}}$ axis.

For the computation of the resultant force $\vec{R}$, we must find the expression of the global matrix of transformation from $(\mathrm{O}, \overrightarrow{\mathrm{i}}, \overrightarrow{\mathrm{j}}, \overrightarrow{\mathrm{k}})$ to $\left(\mathrm{O}^{\prime \prime}, \overrightarrow{\mathrm{f}}, \overrightarrow{\mathrm{d}}, \overrightarrow{\mathrm{e}}\right)$ systems. In this case, we will consider only the rotations between systems (no translations). This transformation will be composed of three rotations.

The first transformation named $\boldsymbol{R}_{\mathbf{1}}$ (figure $02 \mathrm{a}$ ) consists on a rotation about the $\overrightarrow{\mathrm{i}}$ axis with an angle $\alpha_{2}$ such as:

$(\mathrm{O}, \overrightarrow{\mathrm{i}}, \overrightarrow{\mathrm{j}}, \overrightarrow{\mathrm{k}}) \stackrel{\boldsymbol{R}_{\boldsymbol{1}}}{\longrightarrow}\left(\mathrm{O}, \overrightarrow{\mathrm{i}}^{\prime}, \overrightarrow{\mathrm{j}}^{\prime}, \overrightarrow{\mathrm{k}}^{\prime}\right)$ with $\overrightarrow{\mathrm{n}}_{\mathrm{i}}^{\prime}=\overrightarrow{\mathrm{n}}_{\mathrm{i}} ; \overrightarrow{\mathrm{n}}_{\mathrm{j}}^{\prime}=\cos \alpha_{2} \overrightarrow{\mathrm{n}}_{\mathrm{j}}-\sin \alpha_{2} \overrightarrow{\mathrm{n}}_{\mathrm{k}} ; \overrightarrow{\mathrm{n}}_{\mathrm{k}}^{\prime}=\sin \alpha_{2} \overrightarrow{\mathrm{n}}_{\mathrm{j}}+\cos \alpha_{2} \overrightarrow{\mathrm{n}}_{\mathrm{k}}$

The second named $\boldsymbol{R}_{\mathbf{2}}$ (figure $2 \mathrm{~b}$ ) is a rotation about the $\overrightarrow{\mathrm{j}^{\prime}}$ axis with an angle $\varphi$ such as:

$\left(\mathrm{Q},{\overrightarrow{\mathrm{i}^{\prime}}}^{\prime}, \overrightarrow{\mathrm{j}}^{\prime}, \overrightarrow{\mathrm{k}}^{\prime}\right) \stackrel{R}{\longrightarrow}\left(\overrightarrow{\mathrm{i}}^{\prime \prime}, \overrightarrow{\mathrm{j}}^{\prime \prime}, \overrightarrow{\mathrm{k}}^{\prime \prime}\right)$ with $\overrightarrow{\mathrm{n}}_{\mathrm{i}}^{\prime \prime}=\cos \varphi \overrightarrow{\mathrm{n}}_{\mathrm{i}}^{\prime}+\sin \varphi \overrightarrow{\mathrm{n}}_{\mathrm{k}}^{\prime} ; \overrightarrow{\mathrm{n}}_{\mathrm{j}}^{\prime \prime}=\overrightarrow{\mathrm{n}}_{\mathrm{j}}^{\prime} ; \overrightarrow{\mathrm{n}}_{\mathrm{k}}^{\prime \prime}=-\sin \varphi \overrightarrow{\mathrm{n}}_{\mathrm{i}}^{\prime}+\cos \varphi \overrightarrow{\mathrm{n}}_{\mathrm{k}}^{\prime}$

The third named $\boldsymbol{R}_{\mathbf{3}}$ (figure $2 \mathrm{c}$ ) is a rotation about the $\overrightarrow{\mathrm{i}}^{\prime \prime}$ or the $\overrightarrow{\mathrm{f}}$ axis with an angle $\alpha_{\mathbf{1}}$ such as:

$\left(\mathrm{Q}, \overrightarrow{\mathrm{i}}^{\prime \prime}, \overrightarrow{\mathrm{j}}^{\prime \prime}, \overrightarrow{\mathrm{k}}^{\prime \prime}\right) \stackrel{R}{\longrightarrow}\left(\mathrm{O}^{\prime}, \overrightarrow{\mathrm{f}}, \overrightarrow{\mathrm{d}}, \overrightarrow{\mathrm{e}}\right)$ with $\overrightarrow{\mathrm{n}}_{\mathrm{f}}=\overrightarrow{\mathrm{n}}_{\mathrm{i}}^{\prime \prime} ; \overrightarrow{\mathrm{n}}_{\mathrm{d}}=\cos \alpha_{1} \overrightarrow{\mathrm{n}}_{\mathrm{j}}^{\prime \prime}-\sin \alpha_{1} \overrightarrow{\mathrm{n}}_{\mathrm{k}}^{\prime \prime} ; \overrightarrow{\mathrm{n}}_{\mathrm{e}}=\sin \alpha_{1} \overrightarrow{\mathrm{n}}_{\mathrm{j}}^{\prime \prime}+\cos \alpha_{1} \overrightarrow{\mathrm{n}}_{\mathrm{k}}^{\prime \prime}$

This allows obtaining the components:

$$
\left\{\begin{array}{l}
\mathrm{V}_{\mathrm{f}}=-\mathrm{T} \cos \alpha_{2} \sin \varphi \\
\mathrm{V}_{\mathrm{d}}=\mathrm{T}\left(\cos \alpha_{1} \sin \alpha_{2}+\sin \alpha_{1} \cos \alpha_{2} \cos \varphi\right) \\
\mathrm{N}_{\mathrm{e}}=\mathrm{T}\left(\sin \alpha_{1} \sin \alpha_{2}-\cos \alpha_{1} \cos \alpha_{2} \cos \varphi\right)
\end{array}\right.
$$

On the other hand, the moment $\overrightarrow{\mathrm{M}}_{\vec{T}}\left(\mathrm{M}_{\mathrm{f}}, \mathrm{M}_{\mathrm{d}}, \mathrm{M}_{\mathrm{e}}\right)$ will be obtained by using the vectorial cross product, such as :

$$
\left\{\begin{array}{l}
\mathrm{M}_{\mathrm{f}}=\mathrm{R}_{2} \mathrm{~T} \sin \alpha_{2} \sin \varphi \\
\mathrm{M}_{\mathrm{d}}=\mathrm{R}_{2} \mathrm{~T}\left(\cos \alpha_{2} \cos \alpha_{1}-\sin \alpha_{2} \sin \alpha_{1} \cos \varphi\right)+\mathrm{R}_{1} \mathrm{~T}\left(\cos \alpha_{2} \cos \alpha_{1} \cos \varphi-\sin \alpha_{2} \sin \alpha_{1}\right) \\
\mathrm{M}_{\mathrm{e}}=\mathrm{R}_{2} \mathrm{~T}\left(\cos \alpha_{2} \sin \alpha_{1}+\sin \alpha_{2} \cos \varphi \cos \alpha_{1}\right)+\mathrm{R}_{1} \mathrm{~T}\left(\sin \alpha_{2} \cos \alpha_{1}+\cos \alpha_{2} \cos \varphi \sin \alpha_{1}\right)
\end{array}\right.
$$

We can observe in expressions (1) and (2) that all internal forces and moments are proportional to the intensity of the punctual force $\overrightarrow{\mathrm{T}}$. For rather low values of the angles of inclination $\alpha_{1}$ and $\alpha_{2}$ such as $\alpha_{1}=9.14^{\circ}$ and $\alpha_{2}=7.93^{\circ}$, the expressions (2) give the results illustrated on the figure 03-a. We can observe that the bending moments $M_{d}$ are approximately three times higher than those of torsion $M_{e}$ and that for any angle $\varphi$ which varies from 0 to $2 \pi$. This enables to remark a priori that all the cross sections of 
filaments THS with sufficiently closer whorls, works essentially in a bending mode. However, the torque moments $M_{e}$ increase very quickly with the $\alpha_{1}$ and $\alpha_{2}$ angles. Indeed, we can note on the figure 03-b, that the expressions (2) gives for rather high angles of inclination such $\alpha_{1}=15.4^{\circ}$ and $\alpha_{2}=27.9^{\circ}$, torques which are rather close to the bending moments. On the other hand, if one doubles the preceding values of $\alpha_{1}$ and $\alpha_{2}$, the maximum values of the torques are seen amplified twice compared to the bending moments.

These results can be also confirmed for $\varphi=\mathbf{0}$, where, according to (2), we can write:

$$
\begin{aligned}
& M_{d}=T\left(R_{1}+R_{2}\right) \cos \left(\alpha_{1}+\alpha_{2}\right) \\
& M_{e}=T\left(R_{1}+R_{2}\right) \sin \left(\alpha_{1}+\alpha_{2}\right) \\
& M_{f}=0 .
\end{aligned}
$$

For this case of $\varphi=0$, let us define $\alpha=\left(\alpha_{1}+\alpha_{2}\right)$. For a variation from 0 to $(\pi / 4)$ of the angles $\alpha_{1}$ and $\alpha_{2}$ such as $\alpha_{1}=\alpha_{2}=\alpha / 2$, we can obtain the results presented in figure 03-c. We can observe the variation of the bending and torque moments in function of the $\alpha$ angle which varies from 0 to $(\pi / 2)$. We observe that the bending moments $M_{d}$ evolves conversely of the torque moments $M_{e}$. Indeed, for small angles, the filament is inflected primarily. While for raised angles, torsion tends to prevail on the inflection.

On the other hand, the total normal stress due to the contribution of the resultant force $\vec{R}$ and the resultant moment $\vec{M}_{\vec{T}}$ on the cross sections is given by the following equation:

$$
\sigma=\frac{\mathrm{N}_{\mathrm{e}}}{\pi \mathrm{r}_{\mathrm{f}}^{2}}+\frac{\mathrm{M}_{\mathrm{d}} \cdot \mathrm{x}_{\mathrm{f}}}{\mathrm{I}_{\mathrm{d}}}+\frac{\mathrm{M}_{\mathrm{f}} \cdot \mathrm{x}_{\mathrm{d}}}{\mathrm{I}_{\mathrm{f}}}
$$

$r_{f}$ is the radius of the cross section which the shape is assumed to be circular. $x_{d}, x_{f}$, $I_{d}$ and, $I_{f}$ represents the point coordinates in the section and the moment of inertia about the $\overrightarrow{\mathrm{d}}$ and $\overrightarrow{\mathrm{f}}$ axis respectively.

For low values of $\alpha_{1}$ and $\alpha_{2}$, the previous expression (3) can be written as :

$$
\sigma=\frac{-\mathrm{T} \cos \alpha_{1} \cos \alpha_{2} \cos \varphi}{\pi \mathrm{r}_{\mathrm{f}}^{2}}+\frac{\mathrm{M}_{\mathrm{d}} \cdot \mathrm{x}_{\mathrm{f}}}{\mathrm{I}_{\mathrm{d}}}+\frac{\mathrm{M}_{\mathrm{f}} \cdot \mathrm{x}_{\mathrm{d}}}{\mathrm{I}_{\mathrm{f}}}
$$

The shear stresses on the $\overrightarrow{\mathrm{d}}$ and $\overrightarrow{\mathrm{f}}$ direction axes are :

$$
\begin{gathered}
\tau_{\mathrm{d}}=\frac{\mathrm{T} \cos \alpha_{1} \sin \alpha_{2}+\mathrm{T} \sin \alpha_{1} \cos \alpha_{2} \cos \varphi}{\pi \cdot \mathrm{r}_{\mathrm{f}}^{2}}+\frac{2 \mathrm{M}_{\mathrm{e}}}{\pi \cdot \mathrm{r}_{\mathrm{f}}^{4}} \cdot \mathrm{x}_{\mathrm{f}} \\
\tau_{\mathrm{f}}=-\frac{\mathrm{T} \cos \alpha_{2} \sin \varphi}{\pi \mathrm{r}_{\mathrm{f}}^{2}}+\frac{2 \mathrm{M}_{\mathrm{e}}}{\pi \mathrm{r}_{\mathrm{f}}^{4}} \cdot \mathrm{x}_{\mathrm{d}}
\end{gathered}
$$

We can observe in the equation (4), that the normal stress $\sigma$ is proportional to the intensity of the applied force $\overrightarrow{\mathrm{T}}$ and depends on the angle $\varphi$. In the sections with $\varphi=0$ angle; we have represented in table 01 the extremes values of the normal stress $\sigma$. These results are obtained for different values of the intensity of the applied force $\vec{T}$ so that: $\quad \alpha_{1}=9.14^{\circ}, \alpha_{2}=7.93^{\circ}, R_{1}=74.5 \mu \mathrm{m}, R_{2}=322.5 \mu \mathrm{m}$ and $r_{f}=24 \mu \mathrm{m}$. We observe that the extremes values of normal stresses are in an opposite sign and their intensities equivalent. The values of the principal shear stresses obtained by (5) and (6) 
are relatively small compared with the normal stresses results. This also confirms that the cross sections of the twin helical spring THS with sufficiently closer whorls, works essentially in a bending mode.

\section{NUMERICAL ANALYSIS BY THE FINITE ELEMENT METHOD}

The geometrical descretisation consists on the modelisation of theses systems either with three-dimensional frame elements or with three dimensional solid elements (such as tetrahedral, prismatic or cubic elements). The filaments are modelled by frame elements. Based on the Timoshenko beam hypothesis, these elements uses a general three-dimensional formulation by including the effects of biaxial bending, torsion, axial deformation, and biaxial shear deformations (Bathe and Wilson, [06]) (Bathe, [07] ) (Batoz and Dhatt, [08]).

Each element is modelled as a straight line connecting two node points $\mathbf{i}$ and $\mathbf{j}$. Each node activates all six degrees of freedom. In our case, the curved objects are divided into multiple straight elements. Both the geometrical and computational precisions can be improved by a high mesh refinement which consists on the increase of the total number of the elements used by the model (Zienkiewicz and Taylor, [09]). Each frame element is loaded by self-weight and loads due to temperature variation. The self weight loading is applied uniformly on the element segment $\mathbf{i j}$ in the gravitational direction.

The computations will be led in the linear thermo elastic domain where the effects of the self weight solicitations and the variation of the temperature have been coupled. However, the physical properties such as Young modulus, thermal dilatation coefficient and yield stress, have been supposed to be constant and independent of the temperature. This is not really the case (Tietz and Wilson, [04]) (Whittenberg and Nathal,[05]). Their taking into account will imply a non material linearity.

The mechanical properties used in calculations are those of the Tungsten filament. They are identical to those used during analytical calculations, with a Young's modulus $E=276 \mathrm{GPa}$, a yield stress $\mathrm{f}_{\mathrm{y}}=235 \mathrm{MPa}$, a specific weight $\gamma=7.6810^{-5} \mathrm{~N} / \mathrm{mm}^{3}$, a Poisson's ratio $v=0.3281$ and a thermal dilatation coefficient $\alpha=810^{-6}$ by ${ }^{\circ} \mathrm{C}$. The loading due to the temperature gradient corresponds to a variation of $2300^{\circ} \mathrm{C}$ for a reference initial temperature of $20^{\circ} \mathrm{C}$. This variation corresponds to the operating temperature of the lamp (Benghanem, [10] ).

For the study of THS filaments, we choose (figure 04) primary whorls of radius $R_{1}=2.6$ $\mathrm{mm}$ and secondary whorls of radius $R_{2}=4.25 \mathrm{~mm}$ with a wire of radius $r_{f}=0.025 \mathrm{~mm}$ of which the overall length is of $1.00 \mathrm{~m}$. In addition, we choose the same angles of inclination as those of the analytical calculation of the figure 03-b results such as $\alpha_{1}=15.4^{\circ}$ and $\alpha_{2}=27.9^{\circ}$. This filament was modelled by 12784 frame elements.

\section{The Vertical Position}

In order to be able to compare with the horizontal position, we suppose that the filaments are embedded at their two extremities. On figure 04, we observe the deformation due to the isolated action of the variation in the temperature. We can note 
that it consists, as for the case of simple helical spring, of dilatation in the perpendicular plane to the longitudinal axis of the filament. On figure 05 , we observe the deformation due to the combined action of self weight and temperature. It consists essentially of a lengthening of the higher part and a contracting of the lower part on the longitudinal and vertical axis. The predominance of the deformation due to the self weight is quasi total. This can also be confirmed by figure 08 and figure 06 . In deed, in figure08, the torques presents the same orders of magnitude as those of the bending moments. These orders of magnitude are about $10^{-5} \mathrm{Nmm}$. They are negligible with respect of those due to the self weight whose are about $10^{-4} \mathrm{Nmm}$ (figure06).

Moreover, concerning the distribution of the internal loads due to the self weight, we note amplification (figure 06 and fig 07) as well as one approach the embedded extremities. It should be noted also a periodic appearance along each primary whorl. In addition, figure 06 shows that the bending moments about the axes $\boldsymbol{d}$ and $\boldsymbol{f}$ are of the same order of magnitude as those of torsion. This result marks a good agreement with the analytical results established for this filament in figure 03-b. However, the simplifying assumptions such as the concentrated force applied at the lower end to represent the weight of the filament (for analytical calculation), and embeddings of the sections of extremities as well as the uniform linear loading (for numerical calculation), are the essential causes of the differences of the shapes curves and their amplitudes. Another result is showed on figure 07. It is about the normal forces $N_{e}$ whose amplitudes are identical to those of the shear forces $V_{f}$ and $V_{d}$. For the same values of inclination angles $\alpha_{1}$ and $\alpha_{2}$, this is also verified by expression (1) in the analytical calculation.

\section{The Horizontal Position}

From figure 09 , and figure 10 , we also observe that the effect of temperature is quasi non-existent for this position. This enables to confirm for all position the negligible effect of the temperature in linear elastic domain.

Moreover, we observe on figure 11 not only a redistribution of the loads compared to the vertical position (figures 06) but also a certain sensitivity of filaments THS with respect to the change of position. Indeed, the amplification of the moments near the supports in horizontal position (figure 11) is more significant than the vertical position. If one considers the bending and torque moments, their maximum values are about 7.8 $10^{-4} \mathrm{Nmm}$ for the horizontal position instead of $4.510^{-4} \mathrm{Nmm}$ for the vertical. In addition, it should be noted that during the change of position, the intensities of the torsion and bending moments had varied proportionally in the case of filaments THS (figures 06 and 11); whereas they varied conversely one of the other in the case of the SHS configuration (Benghanem et al [02]). Concerning the evolution of the shear and normal forces, we note on figure 12, that their distribution had practically preserved the same shape and the same intensities as for the vertical position (figure 07). We can conclude that only the torque and bending moments are primarily concerned with the effects of the changes of the positions. This highlights a clear sensitivity to position changes for THS filament case.

\section{The Stresses Distributions}

The calculation of the normal stresses is done in the elastic domain according to the expression (3). The checking of these stresses is based on the following inequality: 


$$
\sigma_{\max } \leq \mathrm{f}_{\mathrm{y}}
$$

$\sigma_{\max }$, is the maximum normal stress in a section and $f_{y}$ a yield stress approximately equal to $235 \mathrm{MPa}$.

Let us define by $\rho$ the ratio:

$$
\rho=\left(\sigma_{\max } / f_{y}\right) \text {. }
$$

On the other hand, by adopting the Von Mises criterion, the checking of shear stresses is based on the following inequality: $\tau_{\max } \leq\left(\frac{f_{y}}{\sqrt{3}}\right)$ with $\tau_{\max }$, the maximum tangential stress in a section.

Let us define the coefficient ratio $\gamma=\tau_{\max } *\left(\frac{\mathrm{f}_{\mathrm{y}}}{\sqrt{3}}\right)^{-1}$.

The figures 13 and 14 give the various values of $\rho$ and $\gamma$ for all the sections of the filament. They illustrate the distributions of the maximum normal and tangential stresses in the filament and this, for the two positions considered. We notice that the horizontal position is more unfavourable on the quasi totality of the filament.

\section{CCONCLUSION}

In this paper, two different approaches have been used. An analytical method has been developed and a numerical finite element model has been used. Although the calculations have been performed in the linear elastic domain, some elements of answers have been obtained. In deed, the results enabled not only to conclude on the complementarities of these approaches, but provided a best knowledge of the distribution of the solicitations, the modes of the deformations, the influences of the temperature and the influence of positions changes.

The analytical calculations showed certain dependence with respect to the angles of inclination of the whorls. In deed, for small angles, the filament is inflected primarily. While for raised angles, torsion tends to prevail on the inflection. On the other hand, because of the problems of instabilities of numerical calculations to neglect embeddings of extremities, and of the complexity of analytical calculations to take them into account, the quantitative comparison between the numerical and analytical results could not be carried out directly. However, from the qualitative point of view, the analytical approach proved to be useful for the analysis and the interpretation of some numerical results. In addition, we numerically noted for horizontal position, the increase of loads and maximal stresses on the cross sections located near the extremities. This marks, for the twin helical spring filaments, a clear sensitivity with respect to the change of position. Indeed, the horizontal position proves to be most disadvantageous for this filament, because torsion and bending moments evolves proportionally. Whereas in the case of simple helical spring; the torsion and bending moments evolves one conversely of the other.

Lastly, we also noted that the effects of the variation in the temperature, for linear elastic behaviour, remain negligible with respect to those due to the self weight. This seems confirming the significant part of the non linear behaviour such as creep as a 
principal cause of the rupture. However, more informations could also be obtained in the case of a nonlinear computations. This must be the subject of future studies.

\section{REFERENCES}

[1] Benghanem; N. Kaoua, S.; Azzaz, M, Calcul analytique et modélisation numérique d'un filament doublement spiralé soumis a une traction uniaxiale. Fourth French Lebanese Conf on Sci of Materials Beirut. (2004)

[2] Benghanem; N. Kaoua, S. ; Becheur A; Azzaz, M.,. Modelisation of thermoelastic linear behaviour of tungsten filaments in simple and twin helical spring configurations. International Journal of Solids and Structures. Vol 44, pp 81598176, (2007)

[3] Timoshenko, S.,Strength of Materials. 3rd ed. New York, NY: Van Nostrand. (1955)

[4] Tietz, T.E., Wilson, J.W.,. Behaviour and properties of refraction metals. Stanford University Press, (1965)

[5] Whittenberg; J.D., Nathal, M.V.,. Elevated / low temperature tension testing. Metals handbook, 9th edition, Vol 8, Mechanical testing. (1985)

[6] Bathe, K. J., Wilson, E. L.,. Numerical Methods in Finite Element Analysis. Prentice-Hall, Englewood. (1976)

[7] Bathe , K. J, Finite Element Procedures in Engineering Analysis. Prentice-Hall, Englewood. (1982)

[8] Batoz, J.L., Dhatt, G.,. Modélisation des structures par éléments finis, vol. 2, Hermès, Paris. (1990)

[9], Zienkiewicz, O.C., Taylor, R. L.,. The Finite Element Method. 4th Edition, Vol. 1, McGraw-Hill, London. (1989)

[10] Benghanem, N.,. Caractérisation de la microstructure de filaments de tungstène par EBSP- Approche théorique du comportement en fluage d'un filament à double spiralage. Magister Thesis USTHB ALGIERS (2001).

\begin{tabular}{|c|l|c|c|}
\hline $\begin{array}{c}\mathrm{T} \\
\left(10^{-4} \mathrm{~N}\right)\end{array}$ & $\begin{array}{l}\sigma_{\max } \\
(\mathrm{MPa})\end{array}$ & $\begin{array}{c}\sigma_{\min } \\
(\mathrm{MPa})\end{array}$ & $\begin{array}{c}\tau_{\max } \\
(\mathrm{MPa})\end{array}$ \\
\hline 5 & 17.21 & -17.75 & 3.85 \\
\hline 10 & 34.41 & -35.49 & 7.71 \\
\hline 20 & 68.83 & -70.99 & 15.43 \\
30 & 103.23 & -106.48 & 23.14 \\
\hline
\end{tabular}

Table 01: Extremes values of normal and shear stresses for $\varphi=0$ 


\section{Figures and tables}

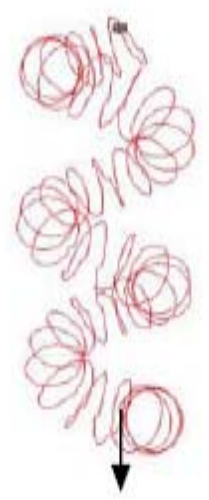

Figure 01: Twin helical spring filament subjected to axial force $\vec{T}$

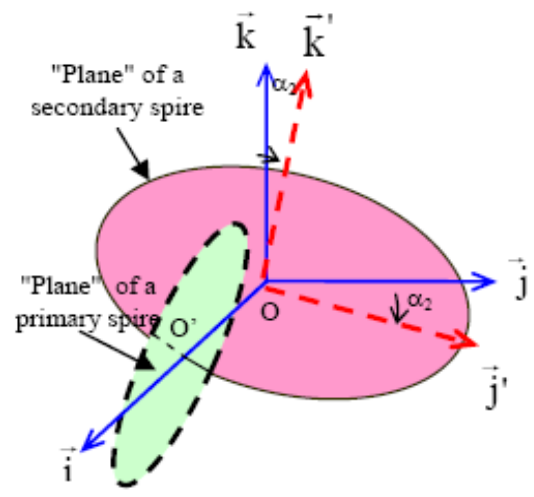

a) Definition of the coordinate systems $(O, \vec{i}, \vec{j}, \vec{k})$ and $\left(O^{\prime}, \vec{i}, \vec{j}^{\prime}, \vec{k}^{\prime}\right)$ Rotation about the $\vec{i}$ axes with an angle $\alpha_{2}$.

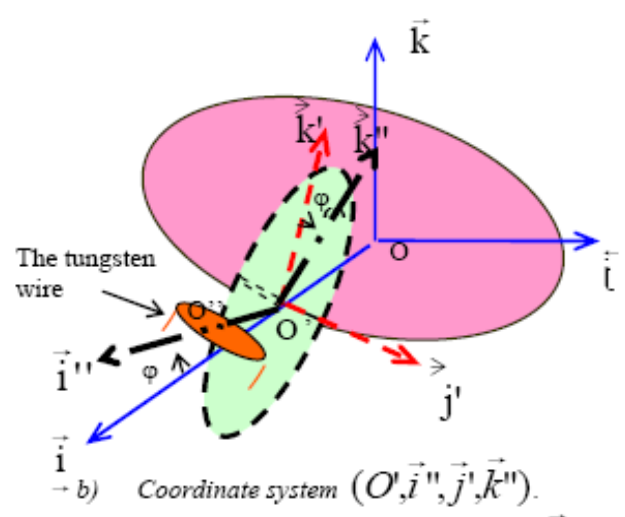
Rotation with an angle $\varphi$ about the $\vec{j}^{\prime}$ axes

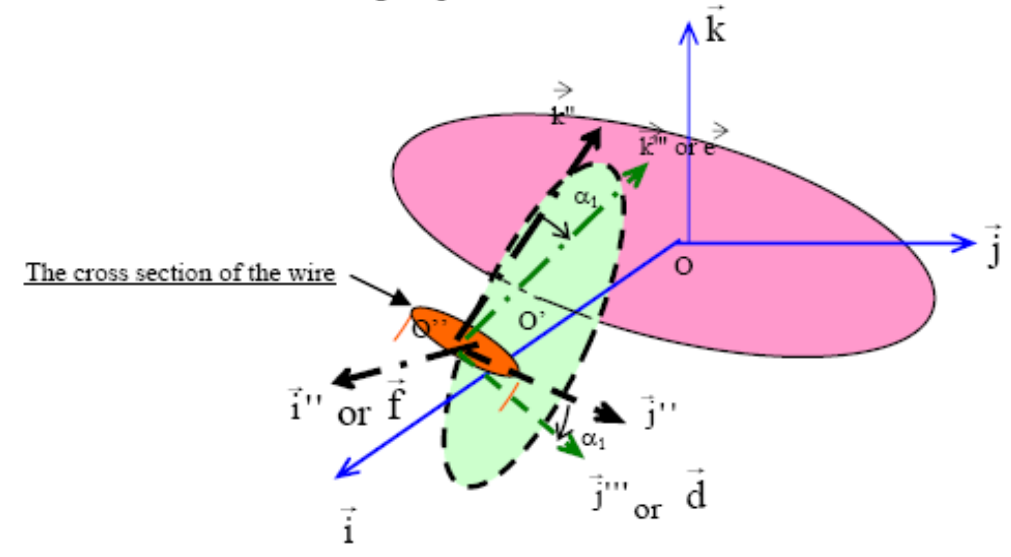

c) System $\left(O^{\prime \prime}, \vec{i}^{\prime \prime}, \vec{j}^{\prime \prime \prime}, \vec{k}^{\prime \prime \prime}\right)$ or $\left(O^{\prime \prime}, \vec{f}, \vec{d}, \vec{e}\right)$ (Rotation with an angle $\alpha_{1}$ about the $\vec{i}^{\prime \prime}$ axes).

Figure $02-$ Definition of the coordinate systems 


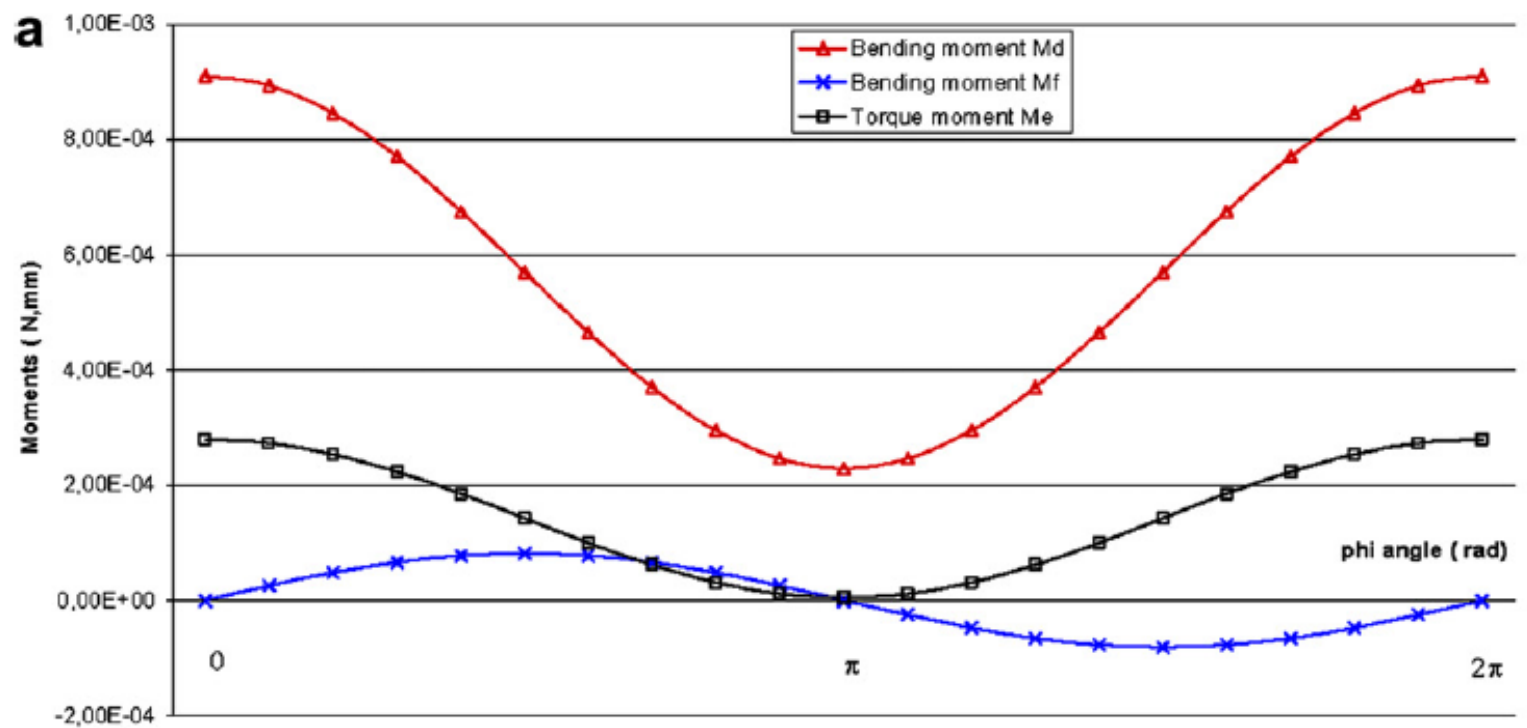

Fig 03-a- Evolution of the components moment $\vec{M}_{\vec{T}}$ in function of the $\varphi$ angle, for T=5 $10^{-4} \mathrm{~N}$ and for small angles $\alpha_{1}$ and $\alpha_{2}$.

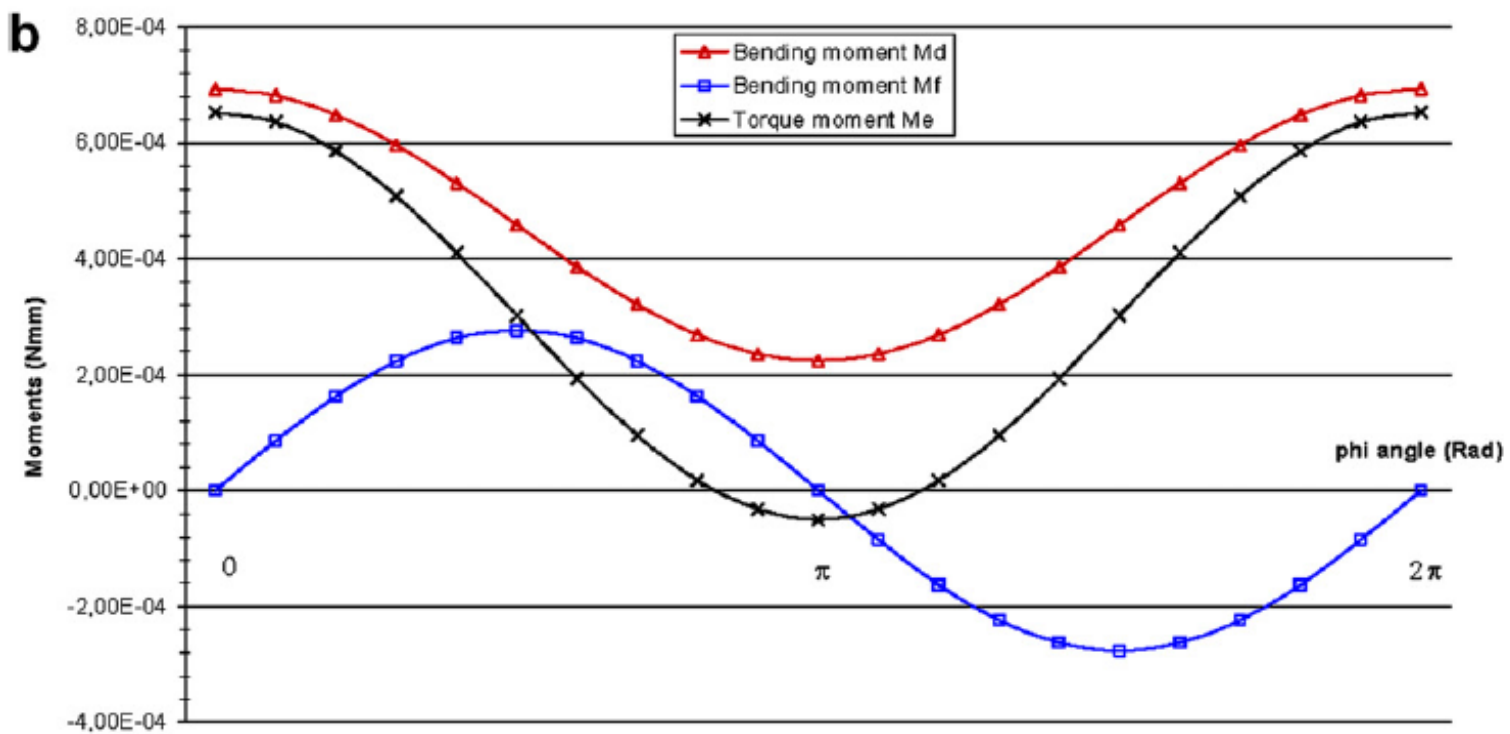

Fig 03-b- Evolution of the components moment $\vec{M}_{\vec{T}}$ in function of the $\varphi$ angle, for T=5 $10^{-4} \mathrm{~N}$ and for enough large angles $\alpha_{1}$ and $\alpha_{2}$. 


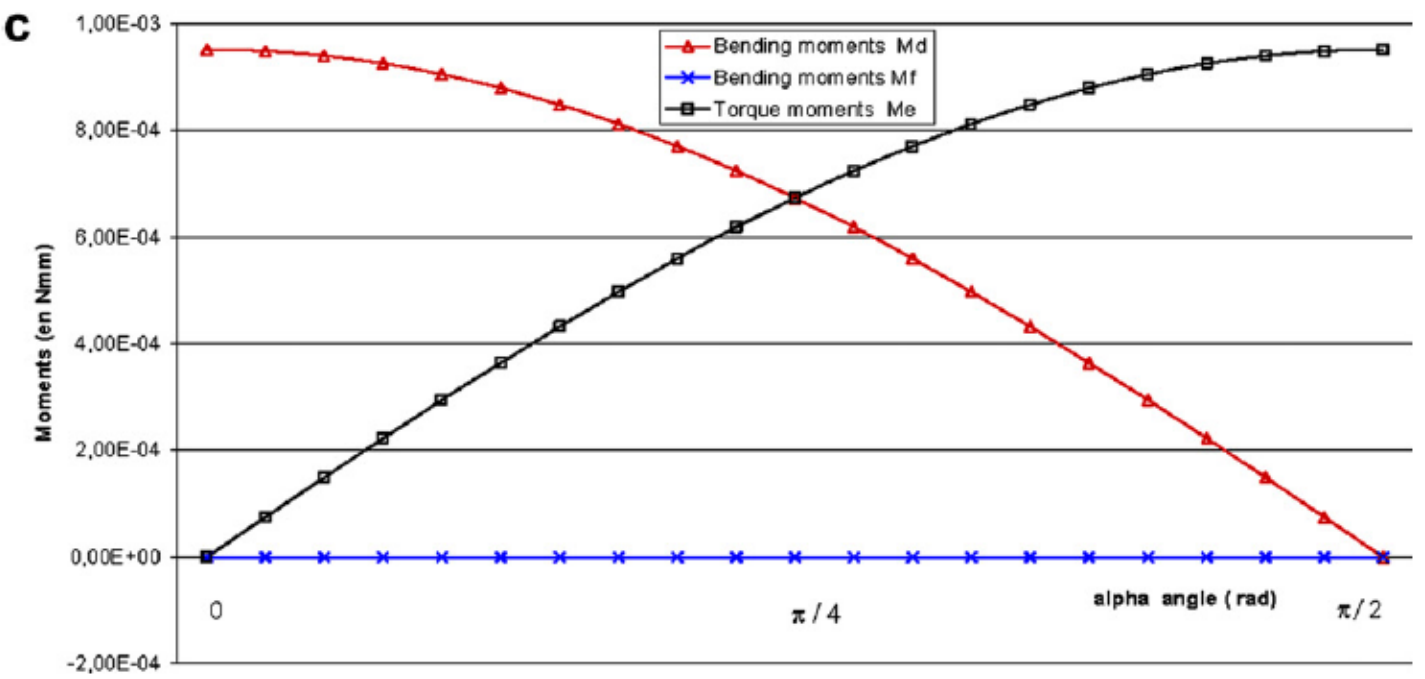

Fig 03-c- Components moment $\vec{M}_{\vec{T}}$ in function of the $\alpha$ angle such as $\alpha=\alpha_{1}+\alpha_{2} ; \alpha_{1}$ and $\alpha_{2}$ varies equally from 0 to $\pi / 4\left(\mathrm{~T}=510^{-4} \mathrm{~N}\right)$.
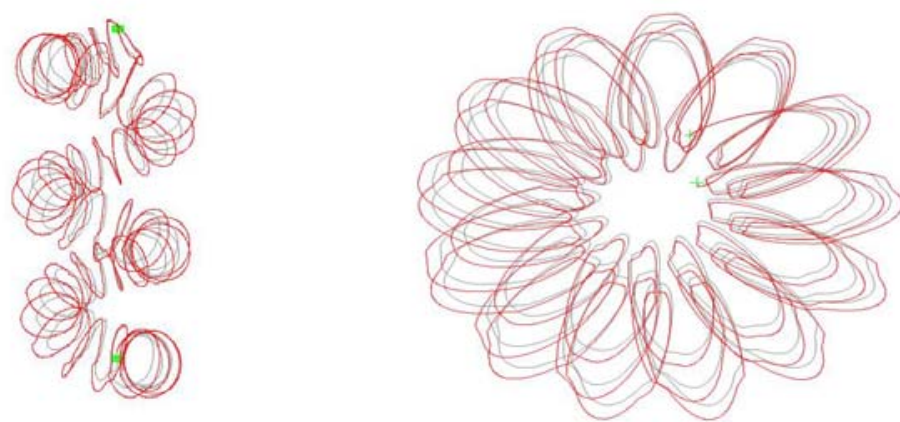

Fig 04: Deformed shape of THS filament under isolated action of temperature (longitudinal and transversal view).
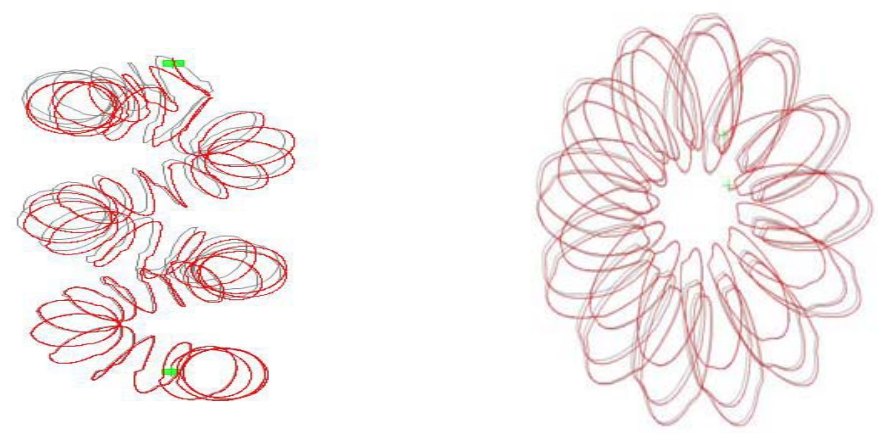

Fig 05: Deformed shape of THS filament under combined action of self weight and temperature (longitudinal and transversal view) 


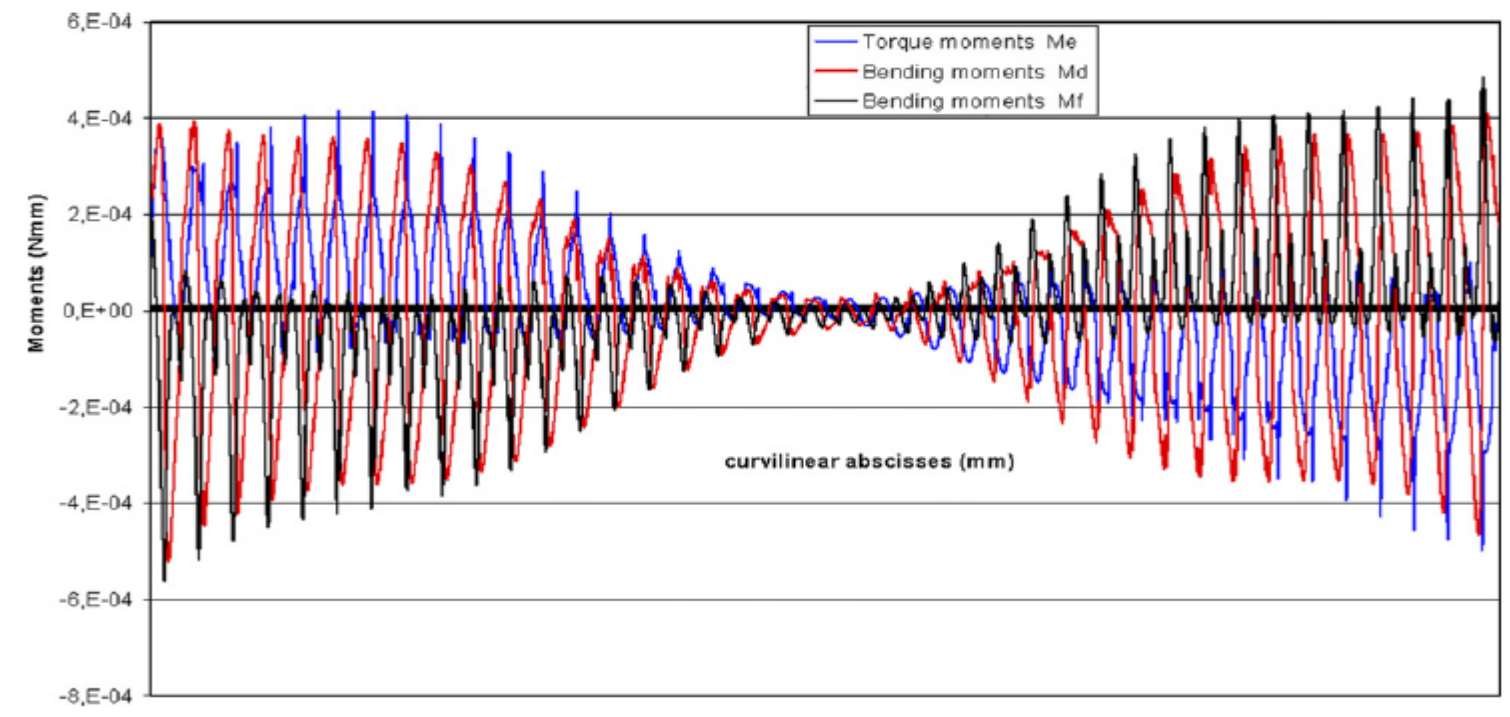

Fig 06 Intensities of the bending and torque moments under self-weight. Filament THS in vertical position embedded at its two extremities.

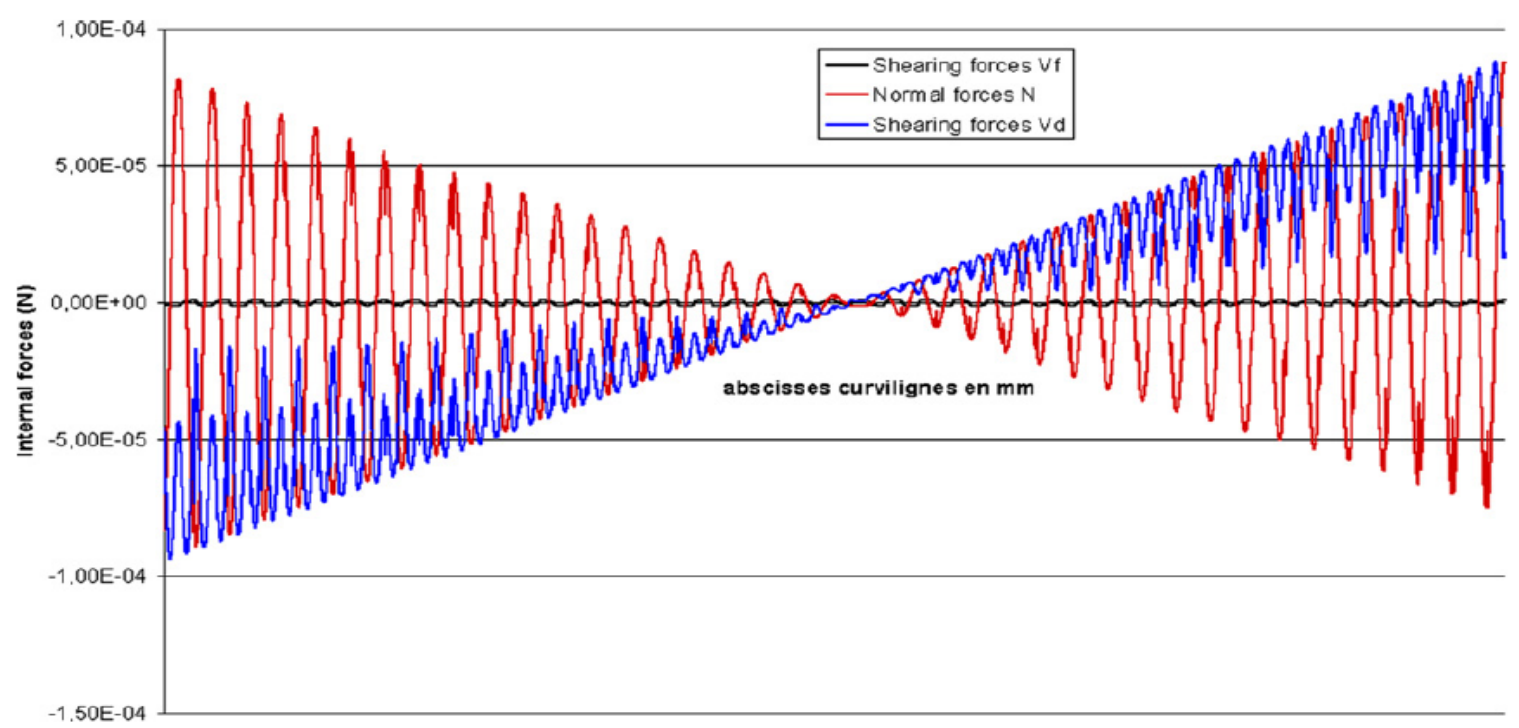

Fig 07: shearing and axial forces distribution under the effect of the self weight of a spring THS embedded at its two extremities 


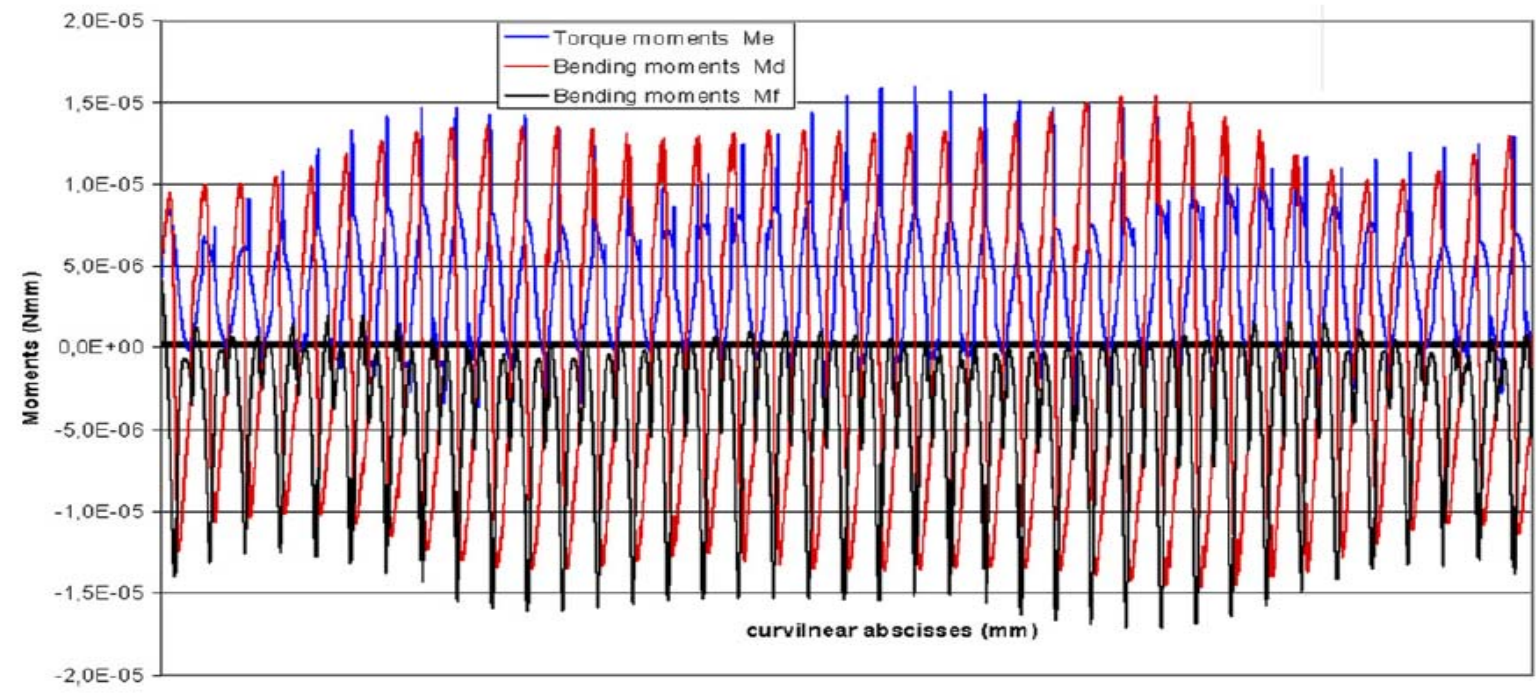

Figure 08: Intensities of the bending and torques moments due to the isolated action of the temperature. Case of a spring THS vertical and embedded at its two extremities.
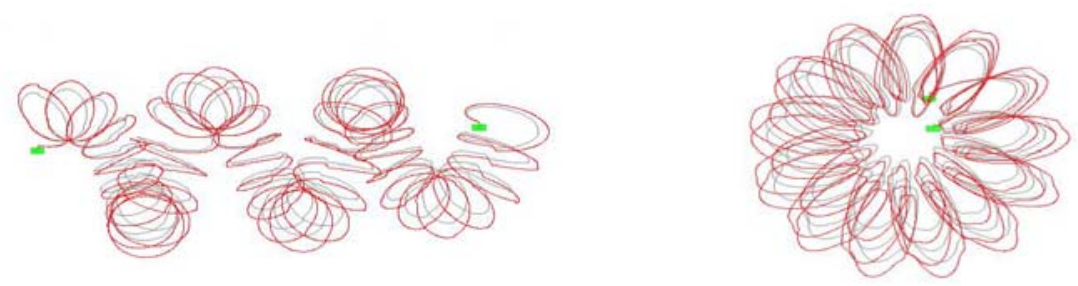

Figure 09: Deformed shape of horizontal twin helical spring THS under only a temperature's variation (longitudinal and transversal view).
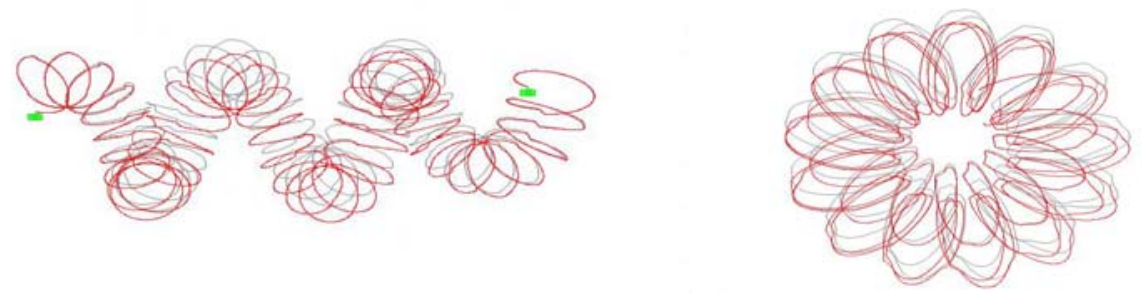

Figure 10: Deformed shape of horizontal twin helical spring THS under self weight effect and temperature's variation (Iongitudinal and transversal view). 


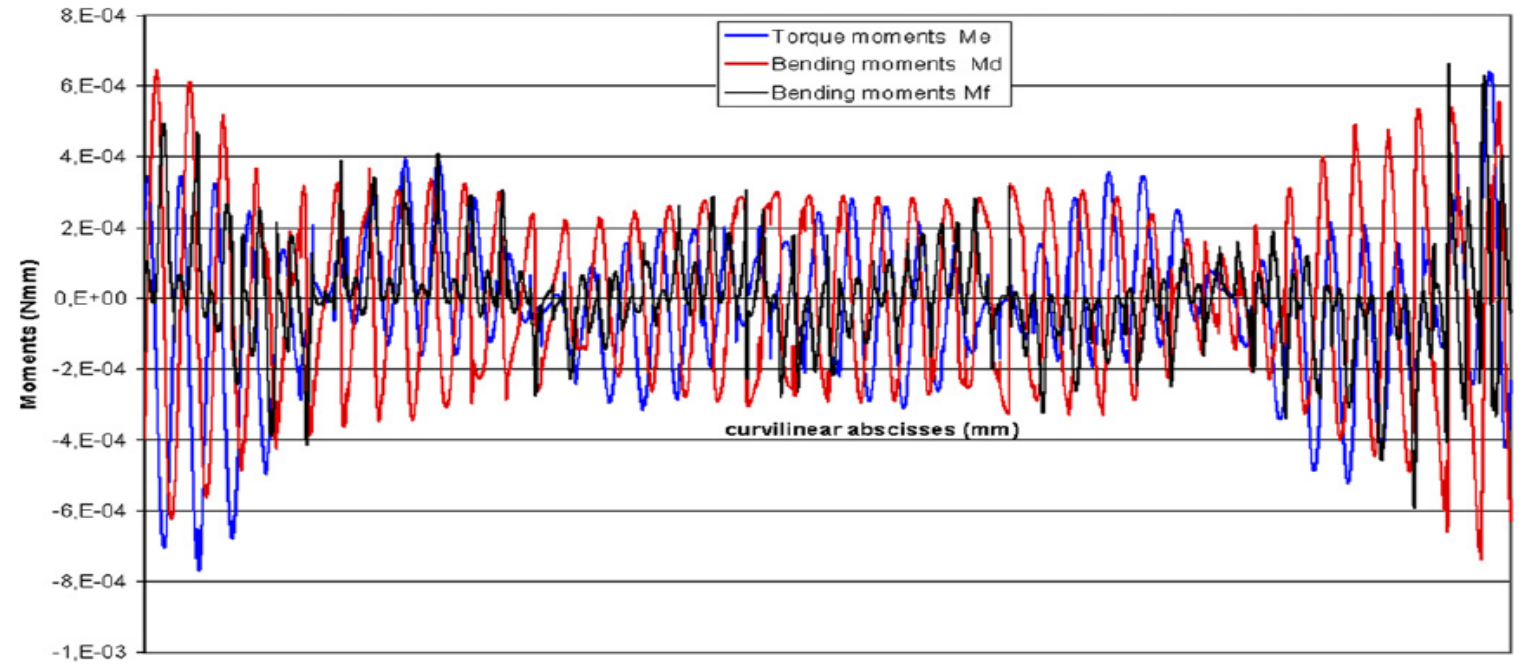

Fig 11: Distribution of torque and bending moments under self weight. Filament THS in horizontal position and embedded at its extremities.

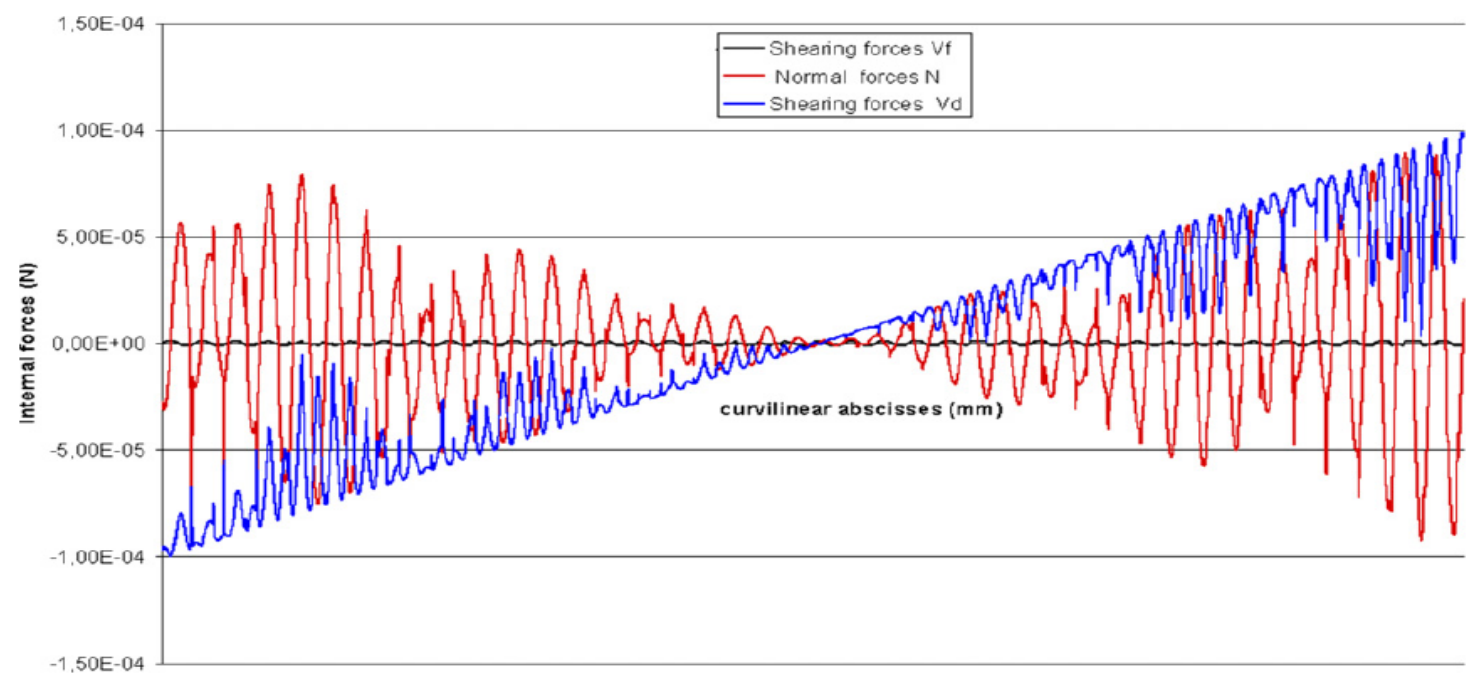

Fig 12 : Shearing and axial forces distribution under the effect of the self weight of a horizontal spring THS embedded at its extremities 


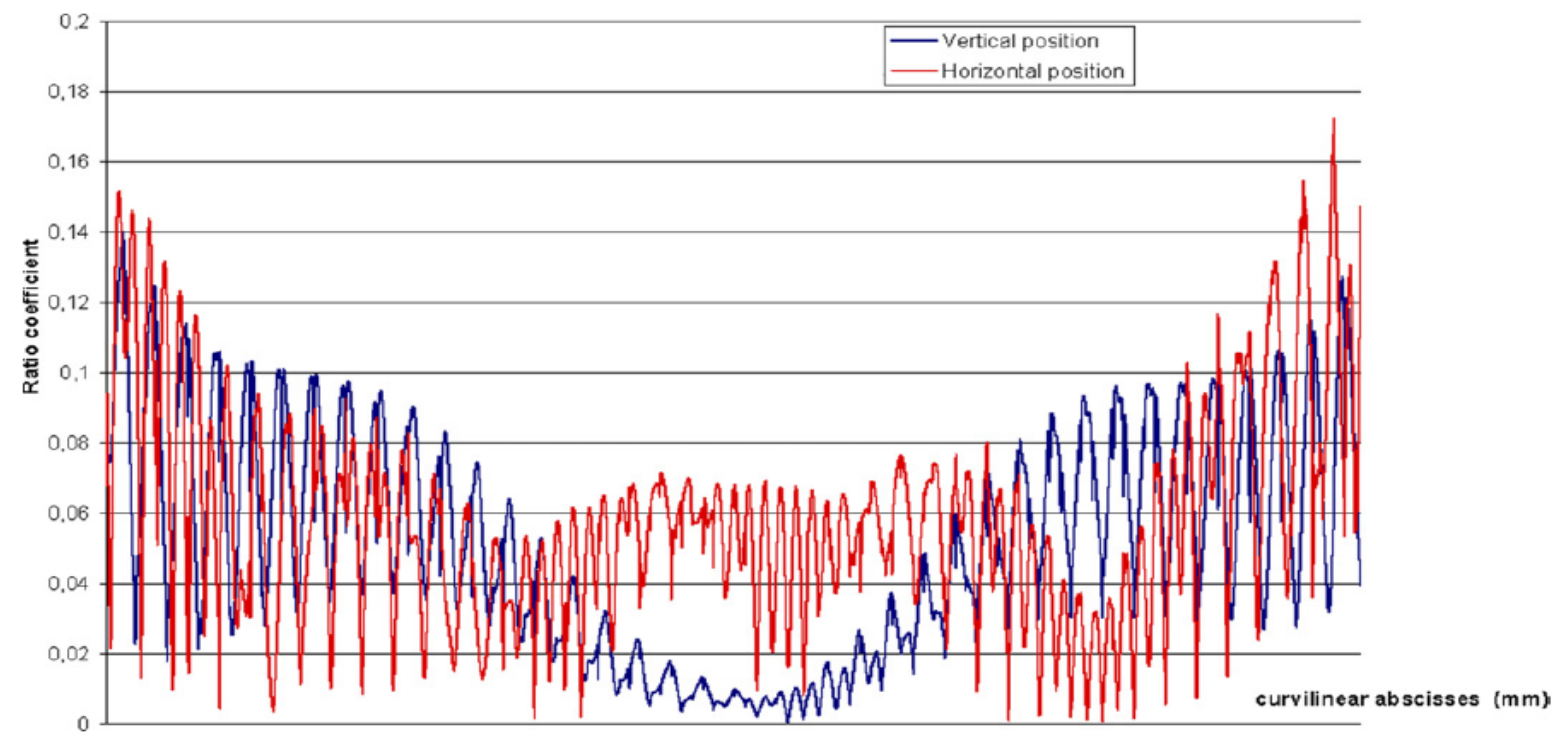

Fig 13 : Ratios coefficient of verification of the normal stresses for horizontal and vertical THS filaments.

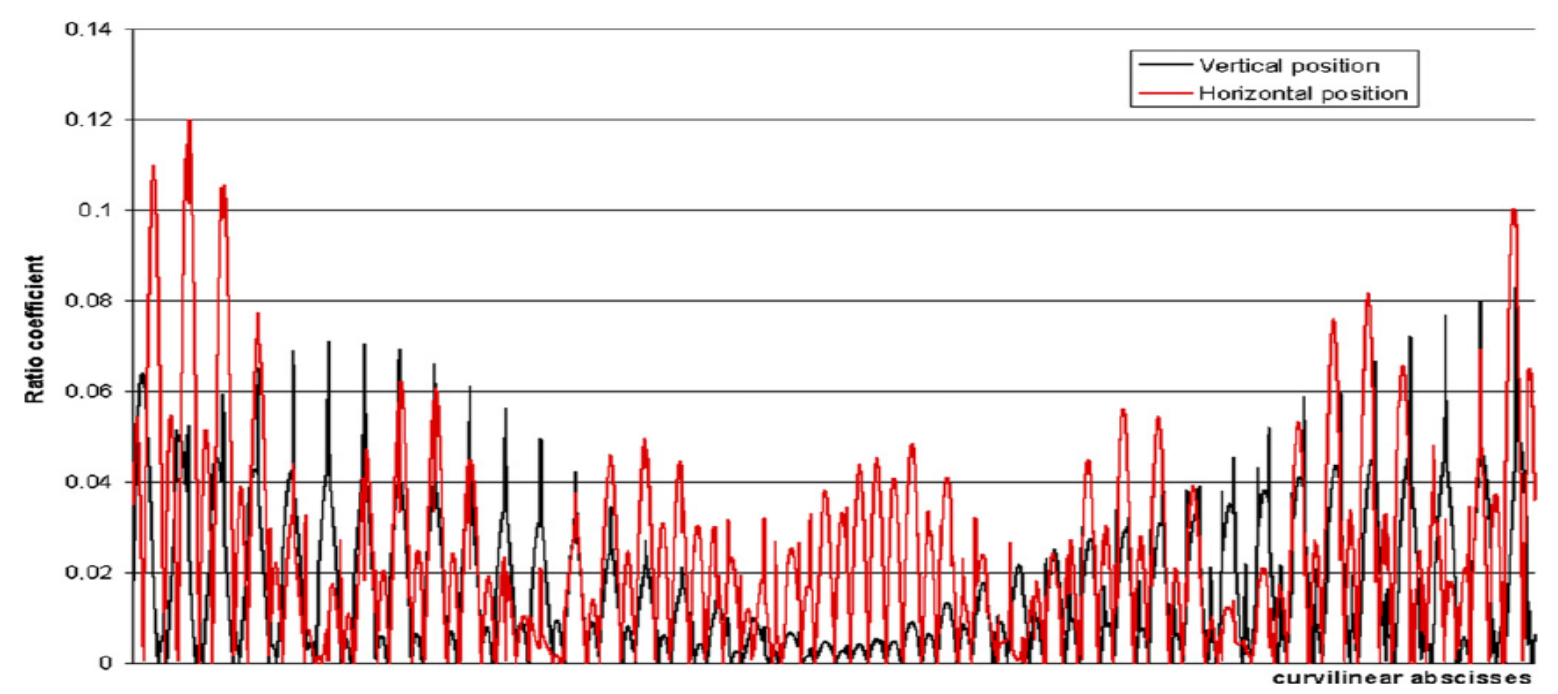

Fig 14 : Ratios coefficient of verification of the tangential stresses for horizontal and vertical THS filaments. 